\title{
An Adaptive Multi-hop Clustered Voting to Improve the Message Trust in Vehicular Ad-hoc Networks
}

\author{
Fatemeh Mirzaee and Mahdi Mollamotalebi
}

\begin{abstract}
The Vehicular Ad-hoc Network (VANET) is used in intelligent transportation systems to make communication between vehicles and make appropriate decisions with regard to road or traffic conditions. Some main research issues in VANETs are unreliability in communication, delay/precision of decision making, information overheads, and handling the nodes dynamicity. This paper aimed to provide an efficient voting method to improve the reliability of decision making for message voting. It also reduces the voting delay and network information overhead. The proposed method is simulated in NS2. The experimental results indicated that the proposed methods decision making precision is improved between $6 \%$ and $30 \%$ compared to similar methods in the literature, under conditions such as traffic amount, number of nodes and operation period time. It also reduces the number of transferred packets between $1 \%$ and $9 \%$ in different environmental conditions.
\end{abstract}

Keywords-Vehicular ad-hoc network, voting, reliability, decision making, precision, packet transfer.

\section{INTRODUCTION}

In order to improve the traffic and reduce the road accidents, the vehicles should be equipped with special means of communication enabling them to exchange traffic status information with other vehicles and roadside infrastructures. Thus, while a vehicle gathers information from the unexpected events, it can help to balance the traffic load. In the event of the occurrence of an accident at the point of the route ahead, the vehicle decides to speed down at the right time, or redirects the route if possible. This could prevent the potential incidence of chained accidents. To this end, the vehicular ad-hoc network (VANET) is used on roads and urban areas [1]-[4].

One of the main issues in vehicular ad-hoc networks is how to communicate between vehicles. For communication in this platform, observer vehicles or available nodes at closer distances to the event, send warning messages to inform nodes away in further distance, so that, they can make the appropriate decision. There may be some unreliable nodes in vehicular ad-hoc networks that attempt to send false information [1]. In recent years, many studies have been done concerning how to manage communications between the vehicles [5]-[9].

Voting is an example of existing methods that assigned a weight to nodes based on geographic proximity to the event in the decision point. Whatever the weight of node is higher, the node in the voting conducted at the point of decision-making has more reliability in terms of accuracy. The disadvantage of this method is that if you rush the vote, some messages have not received while covering important information for

Manuscript received September 16, 2017; revised January 15, 2018.

Fatemeh Mirzaee and Mahdi Mollamotalebi are with Dept. of Computer, Buinzahra branch, Islamic Azad University, Buinzahra, Iran e-mail: motalebi@gmail.com. decision-making at the point of decision. Also in case of delay in voting, received messages may increase at the point of decision, while all of them are not reliable. This leads to an increase in the processing rate of decisions and make potentially inaccurate decisions [10].

In the second section of this paper, we have reviewed the preceding studies in the field of VANET reliability. In the third section, a new method is presented to improve the performance of voting method in detail. In the fourth section, the simulation results of proposed method and comparison with recent similar methods are presented and discussed to evaluate the achievements of current research. Finally, the fifth section of this paper concludes the research report.

\section{LITERATURE REVIEW}

In recent years, many studies have been done concerning how to manage communications between the vehicles [5]-[9]. In the provided multi-agent systems [9], trust management is based on the nodes' behavior history. In this system, the decision maker nodes, estimate trust or uncertainty of messages based on conversations that they had in the past. If the previous conversations with some of the nodes have been satisfactory, the node's trust value for the next conversations increases, and if it is detected as an unreliable node which has sent false information in the previous conversations, its trust value is reduced in subsequent communications. In these systems, issues such as network packet overhead (including geographical and temporal information about the event and the event type), the existence of information overload which needs a high degree of knowledge about the environment, and a single observer are some of the most important weaknesses [1], [9].

Another example of trust management message systems is based on the existence of several base stations to transmit warning messages. Event monitoring nodes send their data packets to the nearest base station in range. Base stations, send data to stations in other areas, so that; finally data packets reach their decision points [11]. The disadvantage of this method is the high cost of implementation and the severe impact of environmental factors on the performance of these base stations.

A voting method for trust management is presented in [10] based on cascading information and nodes influence [10]. In this method, a weight is assigned to each node based on its distance from the event position. As the distance between the node and the event position increases, lower weight is considered for the node selection. Decisions made by a node are affected by other nodes. Our proposed method is based 
on the approach outlined in this research. From now on, this method is called for short ICOV, in the present paper. ICOV method suffers from some problems. Since the implementation of the voting is done at the destination node, a long time is spent on sending data packets, from the node sending the message to the destination node. Therefore, transmission of data packets will be delayed. The rush to vote may cause loss of important messages from the event. Also, high delay in voting may cause other problems such as increased number of false received messages by nodes, which could adversely affect the voting. On the other hand, increasing the number of messages in voting results in more overhead and reduces the reliability and accuracy of voting. This study suggests applying clustering techniques in order to improve the performance of ICOV, in terms of overhead, accuracy, transmitting delay in packets and throughput.

One of the most effective approaches in vehicular ad hoc networks is to classify/cluster the nodes. Different clustering algorithms are provided and they attempt to present appropriate topology for nodes distribution, in order to improve decision-making of the routing between nodes. Some of the existing clustering algorithms in vehicular ad-hoc networks are introduced in the following.

MOBIC [14] is provided for clustering in wireless ad-hoc networks. In this method, the main clustering criterion is based on the mobility of nodes and signal strength. The information of mobility on the transmittance area is shared by sending "HELLO" messages. The node with lowest Mobility is elected as cluster head and the rest of the members are considered as ordinary nodes. The main disadvantage of this method is its low degree of scalability.

Clustering algorithm proposed in [15], is appropriate for urban environments. Clustering formation criterion in this algorithm is based on the predicting movement path of cluster nodes. This algorithm is applied in paths with different directions such as intersections. Nodes in similar movement path of cross roads are classified in the same cluster. In order to cluster in each direction, a cluster is formed before the intersection based on the average of nodes' radio range. As soon as each node reaches a range in which a cluster is present, it sends the "HELLO" message to join the cluster head. If the applicant node does not receive a response from cluster head, it is introduced as cluster head; otherwise, the applicant node will be recognized as an ordinary member. One of the disadvantages of this algorithm is the inability of cluster head to keep track of the nodes which are outside the cluster area. For this reason, the accuracy of the algorithm is reduced in the areas with large number of nodes.

The approach presented in [16], reduces the mobility dependence and distance between the cluster head and member nodes. It is a motion-based distributed clustering approach which has paid a lot of attention to clustering stability. The cluster stability means that cluster head and members maintain in their roles for a long time and cluster head does not change repeatedly. Clusters criteria formation includes responsiveness and sharing accessibility parameters between neighbor nodes. Each of the nodes makes decision about clustering method independently. Because of the similarity of nodes to evaluate current and future data location, data are provided via GPS. Due to the difficulty of this algorithm in identifying the best evaluation parameter to determine the cluster head, it is not capable of providing the optimal solution in clustering.

A resistant adaptive dynamic clustering has been proposed in [17] that considers parameters of speed, location and movement direction. The data are obtained through GPS or similar services. Each cluster head attempts to communicate with the close neighbors and create a continuous chain of clusters. The nodes close to a cluster head are not necessarily members of that cluster. This property comes with multi situation of nodes where, cluster head can be an ordinary member in another cluster. With this feature, routing between clusters is done only through the cluster heads. To be informed of the continuous changes that occur in the network and determine precise routing in the communication area, each node keeps a list of neighbor nodes within its communication range.

The algorithm presented in [18] acts based on criticality in a distributed environment. The critical points are used in forming the large clusters and they provide a high degree of connectivity. If two cluster head are in the same range for a short time, clustering would be postponed. By this manner, unnecessary clustering is prevented. The performance of this algorithm would be adjustable in cases such as reducing the clustering density, reducing the overhead of broadcast messages, using effective capacity of links and reducing the complexity of presenting published messages on the network.

A random clustering algorithm is presented in [19] attempting to do rapid formation of clusters and cluster optimization in maintenance phase. Due to the nodes limitations in sending message, this algorithm does not use multi-hop clustering. To increase the performance, it does not use any location services, so it considers no optimizing for the motion pattern of nodes, which leads to increase stability and longevity of cluster head. On the other hand, this algorithm suffers from interference problem and unnecessary formation of clusters. Cluster interference happens by sudden link disconnections of cluster heads, and creation of unnecessary clusters results by high dynamicity and interaction of neighboring clusters' network messages.

The proposed solution in [20] tries to handle the clustering based on multi-channel communications with the aim of message safety, instant nodes communications and quick data delivery. Clustering parameters are based on nodes' average speed, average life expectancy for the cluster head selection, nodes' direction, and suitable size for clustering. They lead to clusters adapted to large-scale environments.

With regard to increasing number of cars that potentially leads to some accidents, [21] proposed an algorithm considering the speed, movement direction, and the location of each node to specify the cluster configurations. The main objective of the algorithm is to reduce the number of clusters and increase each cluster stability and longevity. The nodes with the same movement patterns are grouped in the same cluster. Clustering starts with low-speed nodes. The position data is obtained through GPS or similar services. In order to select the cluster head, the node having minimal average speed and distance with its one hop neighbors, and also having 
the longer communication with other nodes is found. The proposed solution in the algorithm improves the quality and sustainability of clusters effectively by avoiding unnecessary re-clustering in the network. It leads to more reliability for the exchange of data packets through the clusters.

The algorithm proposed in [22] is used in environments with high mobility nodes. The clustering is based on the node movement speed. The clustering criteria are based on location, direction and nodes speed. One of the weaknesses of this approach is that, if a node speed is different from other members of the cluster, it will be removed from the cluster. Due to not receiving messages from removed node, some of basic information from cluster and network will be lost. This also causes overhead during removing or reconnecting the cluster nodes. In the presence of two clusters with different information in the same area, a cluster head with fewer members will be deleted and all its members should join another cluster head. For this reason, the superior cluster head attempts to send join message to connect to the newcomer's nodes that leads potentially to higher overhead in the network.

Clustering criteria in [23] are based on path detection of nodes handled by digital maps. The cluster head is chosen considering the most traffic flow. Each node calculates propagation levels of the cluster head and sends it in an identified area with velocity and location parameter. If a receiving message node is the member of another cluster, the second node is replaces as the cluster head in terms of superiority. This algorithm provides high efficiency but it has some limitations on identifying the nodes path.

The algorithm PassCAR [24] uses link reliabilities, durability, and tolerability as basic criteria to create a cluster for efficient and reliable routing. This algorithm has three phases as the path traversal, rout reply, and data transmission. In order to increase the probability of path traversal success and reduce the rate of packet loss, PassCAR uses degree of nodes as a criterion for routing. Each node sends "HELLO" messages to its one-hop neighbors periodically. The degree of a node is equal to the number of "HELLO" messages that each node receives. To reduce the number of clusters and avoid creating clusters with weak links, PassCAR algorithm selects a node with the highest degree, on sending the packet.

According to the above mentioned cases, there are several methods for increasing the reliability of the propagated messages, and consequently, improving the quality of wireless ad-hoc networks communication. In this section, a number of trust management methods are introduced for broadcast messages by the vehicle to make a proper decision regarding the occurred event on the roads. The literature review suggests that voting based methods such as ICOV, act better in terms of increasing accuracy in decision making, however, they do not have significant efficiency in terms of time and validity of messages, because of making decisions at the decision point. In order to improve the trust level of messages, clusteringbased methods are introduced that are able to well manage the network communications overhead. The present paper provides a voting based method aiming to reduce the overhead, reducing the sending messages delay, and improving the accuracy of decision making.

\section{PROPOSED METHOD}

The proposed method in this paper called as Adaptive Multi-hop Clustered Voting (AMCV) aimed to improve the reliability of message notifications received by the destination nodes. This method attains its aim by clustering, reducing network communication overhead and increasing the accuracy of decision making in voting. It uses some parameters such as movement direction (two-way road with almost 3 lines in each way), speed (30 to $60 \mathrm{~km} / \mathrm{h}$ ), node location according to meters ([x,y] equals to minimum $[0,0]$ and maximum[582,482]), and type of nodes for priority definition (such as normal cars, police, fire fighters, ambulance and etc.) for clustering. To describe AMCV, a two-way road is considered in Fig 11. The black colored nodes represent nodes along the event direction; the orange ones represent cluster heads; the blue ones are nodes in the opposite direction of the event; the green ones are gateways, and finally the red one represents destination node.

According to Fig. 1. by clustering the nodes that are close to the accident position, the messages voting takes place within each cluster, and then voting will be done between the existing cluster heads at the closest distance from the event point. After selecting the best cluster, the reliable message selected from the voting operation is sent by cluster head to the destination node. By this way, it is expected that appropriate clustering and weighting of nodes reduce the voting process time.

There are two different phases as cluster formation and appropriate cluster selection. In the first phase, finding the appropriate cluster head, forming the cluster, cluster maintenance (including operations such as removing nodes from the cluster, adding nodes to the cluster and re-clustering), and gateway node selection are handled. In the second phase, the selection of appropriate clusters based on criteria such as distance, priorities of each node and movement direction of nodes is done. In this phase, the top cluster is chosen among the existing clusters and then a final message will be sent to the destination node.

Each node sends information of speed, direction, location, total distance from neighbors, type (normal car or aiding services such as police, ambulance, firefighter, etc.) to all its neighbors. After identifying the neighbor nodes and considering criteria of cluster formation, the nodes select the cluster heads $(\mathrm{CH})$ and the gateway node. When the clusters

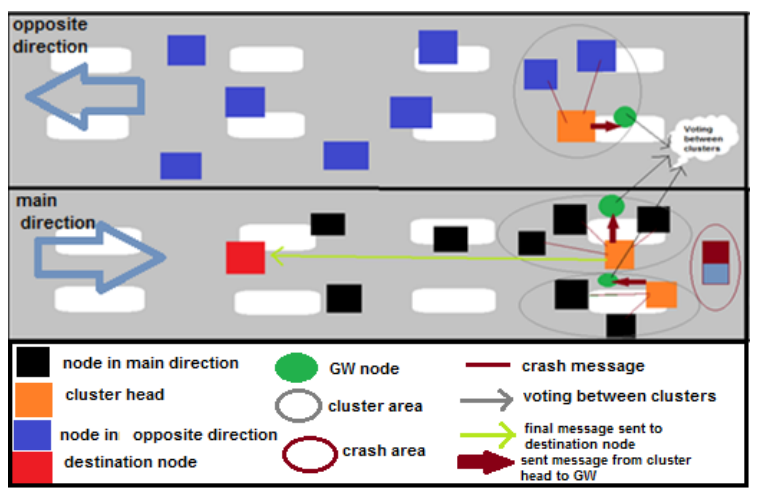

Fig. 1. AMCV method with clustering. 
are formed, each cluster communicates to other ones by its $\mathrm{CH}$. The details of the above two phases is presented in the following.

\section{A. The first phase: cluster formation}

This phase handles communication between nodes, choosing the $\mathrm{CH}$, linking the nodes to $\mathrm{CH}$, and finding the supporting nodes. First, all nodes try to make communication with each other through beacon message transfers. Each node sends beacon message to all of its neighbors. This message contains the information of speed, direction, location, link degree, average of distance difference with neighbors, and average speed difference with neighbors. The nodes detect their one-hop and multi-hop neighbors and obtain their information. After forming the cluster, all nodes in the cluster are considered as a candidate for $\mathrm{CH}$. The process of choosing $\mathrm{CH}$ is shown in Fig. 2

According to the process shown in Fig. 2, the node with higher priority introduces itself as $\mathrm{CH}$ to other neighbors. It will be approved by all nodes based on criteria such as distance, speed difference and neighbor's communicational degree. After choosing $\mathrm{CH}$ in each area, $\mathrm{CH}$ sends a message containing location information, speed, and degree to its neighbors to introduce itself to nodes within the signaling range. As each of neighbors receives the $\mathrm{CH}$ message, they send a connection request message to $\mathrm{CH}$. The $\mathrm{CH}$ accepts the nodes request as cluster member based on their distance and speed. It accepts a certain number (maximum 20) of membership requests on the basis of network environment density. As a result, if a new node has requested membership in the cluster after the completion of the cluster capacity, due to increased messages exchanged in clusters, each cluster increases the voting duration that would reduce the decision accuracy. Hence $\mathrm{CH}$ issues rejection message to the requesting nodes as a response. After the formation of clusters, $\mathrm{CHs}$ send information update request to the members for updating the cluster data. The members reply their new information (speed, direction, location, degree, average of difference distance and speed from the neighbors) to $\mathrm{CH}$. To handle the inter-cluster communications, the closest node to border of the cluster is chosen as gateway.

In dynamic conditions, the nodes may exit from network at any time. If a node receives no message from its neighbors during a certain period (around 10s), it assumes itself as independent of all the clusters and begins the re-clustering process. Also, if $\mathrm{CH}$ doesn't receive any message from a member node after this period, it removes the node from the membership list. Lack of messages may be caused by getting away from the signal range.

\section{B. The second phase: appropriate cluster selection}

In this phase, choosing the messages of a cluster for participating in the voting operation is handled. According to III-A clustering is done based on common characteristics like direction, distance, and speed difference. For example, two nodes with the same distance to an event position are selected for inclusion in a same cluster. Also the nodes in the same movement direction and the same movement speed have the ability to fit in the same cluster. In the proposed method, the appropriate cluster selection for voting operation takes place based on the vicinity criteria of $\mathrm{CH}$ to the source event. Vicinity means to have minimum distance of considered node to the event position. The lower distance of an event and its observer node, the cluster nodes have a greater chance of being selected in voting process. So first, for each cluster, it is considered a possibility based on the type of $\mathrm{CH}$ (regular nodes or aided service nodes) and distance $(d)$, that this possibility is considered as $1 / d$. As $\mathrm{d}$ is short and also $\mathrm{CH}$ is one of particular types (aided services node like police cars), its chance is higher being participated in the voting operation.

Node direction is another necessary parameter for proper clusters selection. If the nodes move in the opposite direction of the event position, they will have a higher chance to choose the cluster for voting among members. The reason is that the vehicles in the opposite direction that are passing through the accident scene are nodes nearing which will arrive to the scene of event (e.g. accident) in next moments. Such cluster nodes, inform cluster nodes in the opposite direction to the event, and thus the transmitting speed of alert messages would be faster and it would decrease the transmitting delay. In order to select the top cluster, the parameters distance, $\mathrm{CH}$ type, and movement direction should be considered.

Equation (1) is used to calculate the priority of clusters:

$$
\text { Priority }=\frac{1}{d} \text { dir } \cdot \text { type, }
$$

where $d$ represents the distance from event position, and dir shows priority directions (that for clusters with the similar direction of event position will be considered 1 . The value 2 will also be considered for clusters with opposite direction). The parameter type specifies $\mathrm{CH}$ type (which is considered as 1 for regular nodes and 2 for aided services nodes). Fig. 3 represents a top cluster selection process based on the above equation.

Once the voting between the cluster heads is completed, a cluster with highest priority is chosen as top cluster and the $\mathrm{CH}$ performs the voting operation between its members as an indicator of that cluster and sends the final result message

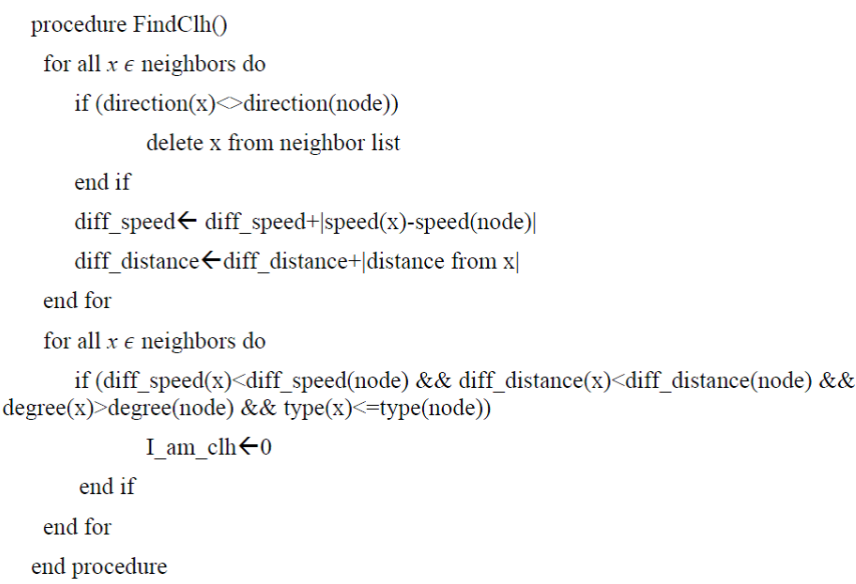

Fig. 2. Cluster head choosing process. 
TABLE I

THE SIMULATION COMMUNICATION

\begin{tabular}{l|c} 
Parameter & Value(s) \\
\hline Data link layer protocol & $802.11 \mathrm{p}$ \\
Routing protocol & OLSR \\
Radio propagation model & Nakagami \\
Transmitter queue's capacity & 60 \\
Queue size control algorithm & Remove from end \\
The size of data link layer header & 416 bit $(52$ byte $)$ \\
The size of the physical layer header & 1351 bit \\
ACK frame size & 304 bit (38 byte) \\
The frequency and time of simulation & 10 times to 200 \\
The type of protocol used for VANET traffic & UDP with CBR \\
\hline
\end{tabular}

to the destination node. This potentially reduces the required time for voting. Also, if it is necessary, the voting can be repeated between the two or more top clusters. In this case, after selecting the best available top clusters near to the event position and performing the voting within the cluster, the obtained results from each cluster will be sent from $\mathrm{CH}$ to gateway of cluster. Then the final voting is done through each cluster gateway.

\section{IMPLEMENTATION AND EVALUATION OF THE PROPOSED METHOD}

In order to evaluate the proposed method, it is implemented in NS2 version 2.33, and the results of the experiments are compared with the recent similar research ICOV [10] in different aspects of network throughput, packet delays, overhead, and the accuracy of decision-making on the event position. The experiments are performed in various scenarios and configurations of the road traffic, the number of events (one to five events per day), nodes' speed (30 to $60 \mathrm{~km} / \mathrm{h}$ ), and two-side roads with at most 3 lanes on each side. The experiment of each configuration is repeated for ten times and the average value of obtained results is reported. In the following scenarios, for transmitting data packets, the intervehicle traffic protocol based on UDP is used. The number of nodes varies on the values 20,30, and 40. The number of clusters is different based on vehicles distance and traffic. Close vehicles (distance between 5 to 50 meters) are included in the same cluster.

Also, based on the node types, they are prioritized in a way that ordinary nodes are given priority 1 and service-providing nodes have priority 2 . Table I indicates the values of simulation parameters.

Some values such as data link layer protocol, routing proto$\mathrm{col}$, and radio propagation model are fixed, and the duration of

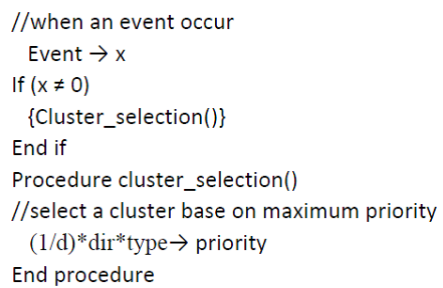

Fig. 3. The top cluster selection algorithm simulation is considered up to $200 \mathrm{~s}$ to increase the accuracy. In order to calculate the accuracy of decision-making and operational overhead factors (2) and (3) are used:

$$
\text { Accuracy }=\frac{\text { true_d }}{\text { whole_d }} \cdot 100,
$$

where true_d is the valid data packets which carried the true data about the events and it is obtained from the most similar parameters of messages that are issued during $0.3 \mathrm{~s}$ in the network, and whole_d indicates the entire transmitted data packets which contains the information about the event.

$$
\text { Overhead }=\frac{e x \_d}{\text { whole_d }} \cdot 100,
$$

where $e x \_d$ is the number of additional data packets in the messages, and whole_d indicates the total number of data packets transmitted on the network.

The latency is measured by clustering duration as:

$$
\text { Latency }=\frac{\text { sum_latency }}{\text { opt }(n)},
$$

where $\operatorname{opt}(n)$ represents the number of nodes participating in the latency time computation and sum_latency is the execution latency at any time. It is the overall delay time in operation and it is based on previous sum_latency and ra_latency (which means the random delay time resulted from any noise such as hard weather condition that cause latency), i.e. sum_latency $=$ sum_latency $+[$ ra_latency $]$. Latency is defined as the delay amount time for each node. The packet throughtput is measured as:

$$
\begin{gathered}
\text { Packet throughtput }= \\
=\frac{\text { I.C.O.V.result-A.M.C.V._result }}{I . C . O . V . \_r e s u l t} \cdot 100 .
\end{gathered}
$$

All the packets carry fields such as maximum distance between node locations, the highest speed difference between nodes, direction in road, speed of nodes, and degree of nodes which defines the number of its neighbors.

The throughput is measured by calculating the number of selected data packets from the top cluster close to the event position, and transmitting to the node in the destination. This indicates the number of transmitted packets at a certain time. In order to send a message and detect nodes' traffic in the network, by starting the simulation in the fixed intervals $(3 \mathrm{~s})$, all nodes start to transmit the probe packets. Then, based on the received responses, the sender nodes identify the network traffic, nodes within their communication range, and perform the clustering process. Over the second period (30 s), they update the information and derive new information about network conditions. This process will continue until the end of the simulation period. The examined scenarios for evaluations are presented below.

Scenario 1: In this scenario, changes are applied on the transmission rate of data packets based on network traffic. The rate of data packets (or VANET traffic) is considered as different forms based on the number of nodes used in the simulation process (the levels of latency, overhead, decision making accuracy, and throughput). Also, CBR data packet 
transmission rate is considered as 200, 500 and 1000 bits per second. Each algorithm is executed during $60 \mathrm{~s}$.

Fig. 4 and Fig. 5 show the obtained results in terms of decision accuracy and throughput of packets respectively. The packets throughput is between 50 and 200 and it only depends on the number of nodes and it is not affected by changing the network traffic.

The reason is that in the throughput calculation, only the effective packets are considered in the final decision, and the amount remains fixed with certain number of nodes. The effective packets refer to the messages transmitted from the nodes near the event position. The number of packets transmitted in AMCV is less because the voting is done on the clusters close to the event position and subsequently choosing only one optimal cluster to send the final message which increases the reliability and accuracy.

In ICOV, the messages related to an event are transmitted based on the following sequence which leads it to have less accuracy and reliability:

$$
\sum_{i \in R 2} d_{i}+\ldots+\alpha^{n-1} \sum_{i \in R 1} d_{i}+\alpha \sum_{i \in R n} d_{i},
$$

The above sequence shows the scaling of nodes in carrying the reliable message, where $R$ represents the number of node steps towards the event position, $d$ is the distance of the nodes to the event position, and $\alpha$ represents the weight of nodes in the environment. Here the step refers to the queue of the nodes versus the event that each row is measured at the certain distance $\mathrm{d}$ from the event position.

Fig. 4 indicates that packets throughput is between 50 and 200 and it only depends on the number of nodes and it is not affected by changing the network traffic. The reason is that in the throughput calculation, only the effective packets are considered in the final decision, and the amount remains fixed with certain number of nodes. The effective packets refer to the messages transmitted from the nodes near the event position. The number of packets transmitted in AMCV is less because the voting is done on the clusters close to the event position and subsequently choosing only one optimal cluster to send the final message which increases the reliability and accuracy.

According to Fig. 5, the accuracy of decision-making in AMCV is improved $12 \%$ to $27 \%$ compared to ICOV. The re- sults indicate that decision making accuracy is more improved in the condition of having low number of nodes (20 nodes). It is because that clustering for fewer nodes is performed faster, and nodes in each cluster have common features. In the conditions of $\mathrm{CBR}=500$ or $\mathrm{CBR}=1000$, and having more number of nodes, the accuracy of both methods AMCV and ICOV is reduced due to higher network overheads. But based on the average results of experiments, AMCV improved at least $12 \%$ which means that it is almost compatible with high-traffic conditions.

Scenario 2: In this scenario, some changes are applied on the presence duration and nodes operations in the network. The presence of the nodes is considered in three different cases. Also, considering different number of nodes for each time period, the latency, overhead and accuracy of decisionmaking is measured in ICOV and AMCV methods. Data packet transmission rate (CBR) is considered as 200 bps and the algorithms are evaluated in three different periods of $50 \mathrm{~s}$, $100 \mathrm{~s}$ and $200 \mathrm{~s}$. Fig. 6 and Fig. 7 show the the packet throughput amount and accuracy of ICOV and AMCV in this scenario.

Fig. 6 represents the throughput of the packets in AMCV and ICOV methods during different periods of time. It indicates that in short operation times and less number of nodes, the throughput of the packets is increased between $1 \%$ and $6 \%$ in AMCV which can be construed as stable rate of changes. Increasing the period length causes more changes in the rate of throughput. In longer time period of operation in ICOV method, the number of transmitted packets is increased over the time. This reduces the accuracy and reliability of the decision making. On the other hand, by applying the clustering on AMCV and voting between the clusters, lower number of packets are selected to exchange data.

According to the results shown in Fig. 7, the accuracy of decision-making in AMCV is increased between $6 \%$ and $17 \%$ compared to ICOV. The results also indicate that if the number of the nodes is low, the accuracy of decision-making in two methods is almost the same. When the time duration of the operation and the number of nodes are increased, the accuracy of decision-making in AMCV increases compared to ICOV. It is because that at longer operation times, the number of nodes

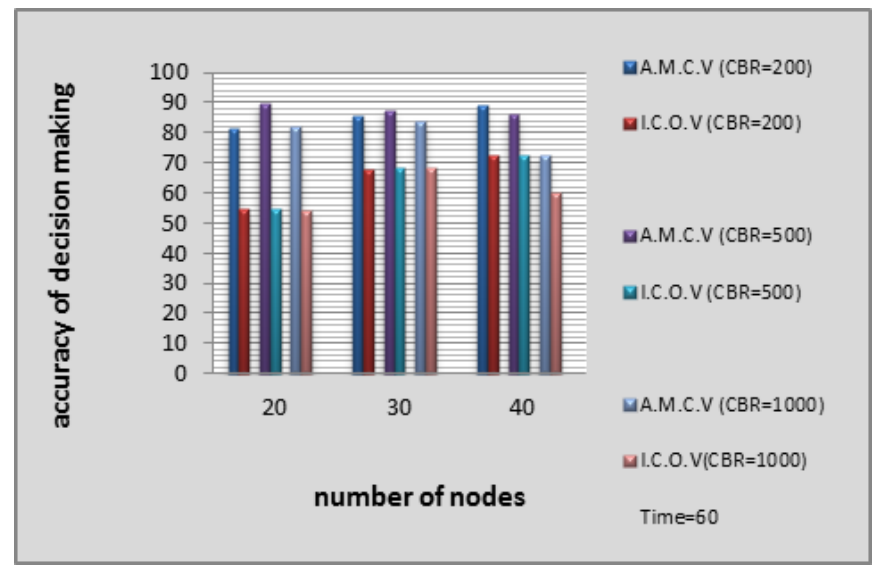

Fig. 5. Accuracy for different traffic rates.

Fig. 4. Packet throughput of decision-making with different traffic rate. 
participating in the operation and the validity of the messages transmitted to the final node is increased by clustering.

Scenario 3: In this scenario, the simulation environment is considered as noisy. It is expected that noisy network environment (in terms of weather conditions or route) lead to changes in measured accuracy, overhead, latency and throughput. The rate of CBR is considered as $200 \mathrm{bps}$ and the time period is assumed as $40 \mathrm{~s}$.

The results shown in Fig 8 indicate that the throughput of packets with the presence of noise in the network environment in AMCV is improved. The throughput of packets is at least $0.5 \%$ when the network is not busy; it is increased up to $4 \%$ when the network traffic increased.

As shown in Fig 9, the accuracy of decision-making in AMCV is increased 5-8\% compared to ICOV. Moreover, the network noise (such as abnormal weather conditions with at least $99 \mathrm{~dB}$ power and $10 \mathrm{MHz}$ bandwidth) potentially prevents some packets to reach destination and it reduces the reliability. In AMCV, clustering operation is done in a certain range and less final data packets are transmitted compared to ICOV; thus the amount of packet loss is less and accuracy of decision-making is higher. The accuracy of decision making in low number of nodes is more improved because the decisionmaking becomes more accurate and with less overheads.

Scenario 4: As the last scenario, nodes are considered with

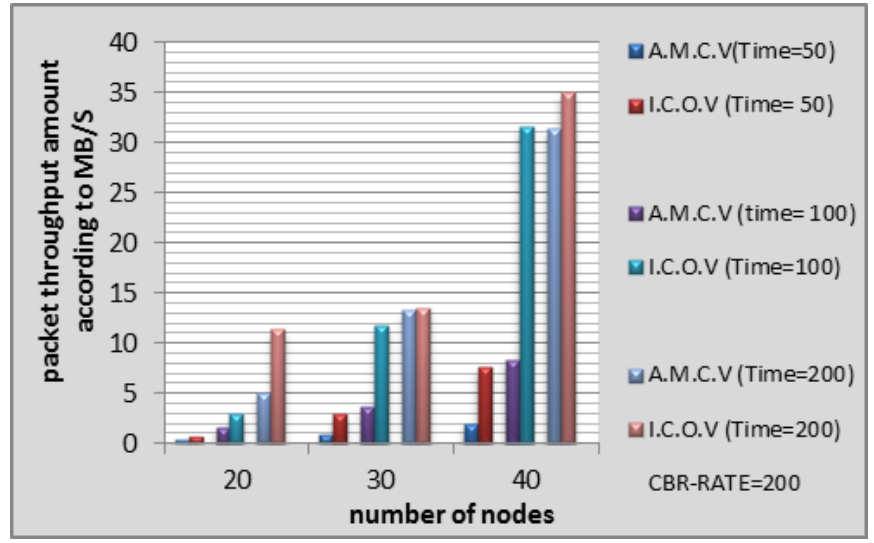

Fig. 6. Packet throughput amount of decision-making in different time and constant traffic rate.

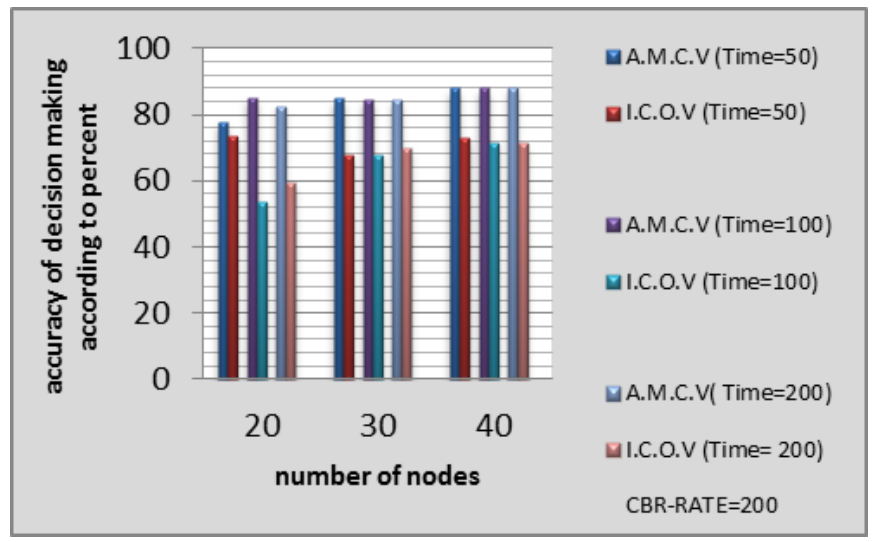

Fig. 7. Accuracy of nodes of decision making in different time and constan traffic rate. different priorities. In this scenario, the rate of transmitted data packets (the network traffic) and the time period are considered as constant. Two levels of priority (regular and special) were randomly assigned to nodes. The rate of CBR is assigned to $200 \mathrm{bps}$ and the execution time period is assumed as $40 \mathrm{~s}$. The priority of the regular nodes and special nodes are set as 1 and 2 respectively. The latency, overhead, accuracy of decision-making and the throughput are measured in ICOV and AMCV methods.

Fig. 10 shows Packet throughput of decision-making amount with different priority for both methods which shows that the transmission packets of proposed method in AMCV is improved to ICOV. With a small number of nodes, packets transmission in AMCV is improved $0.5 \%$ at least. As the number of nodes increase, the amount of packets transmitted during the execution period is improved at most to 5\% compared to ICOV. This indicates that with regard to the priority of the nodes, the number of packets transmitted during simulation is reduced in AMCV. Thus AMCV presents an acceptable level of accuracy detection and flexibility.

The results shown in Fig. 11 indicate that the accuracy of decision-making in AMCV is increased between $7 \%$ and $40 \%$ compared to ICOV. Moreover, by having more regular nodes

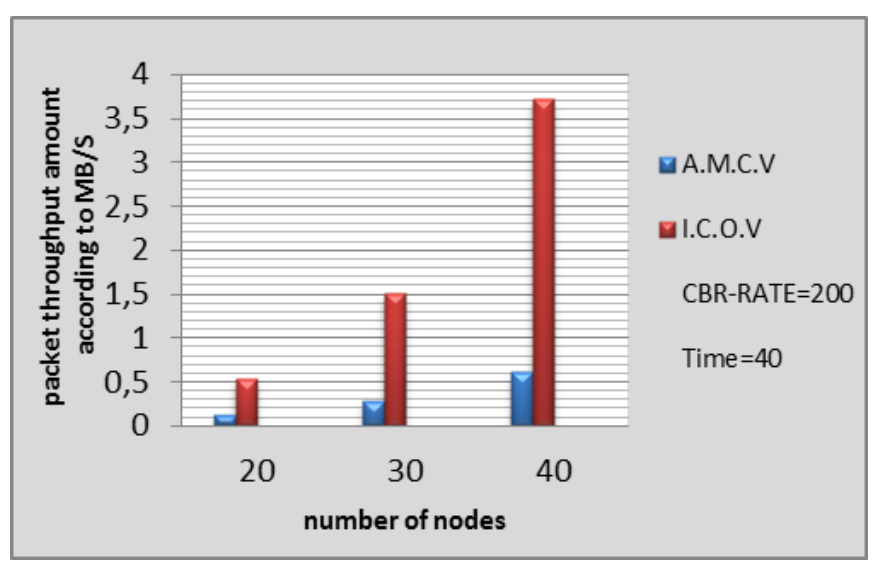

Fig. 8. Packet throughput amount of decision-making with noise in AMCV and ICOV methods.

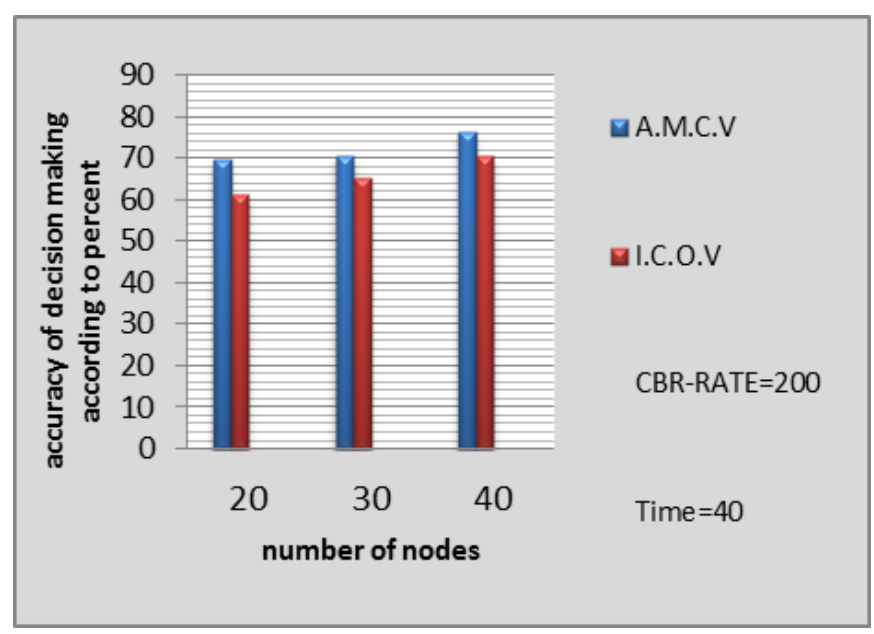

Fig. 9. Accuracy with noise in AMCV and ICOV methods. 


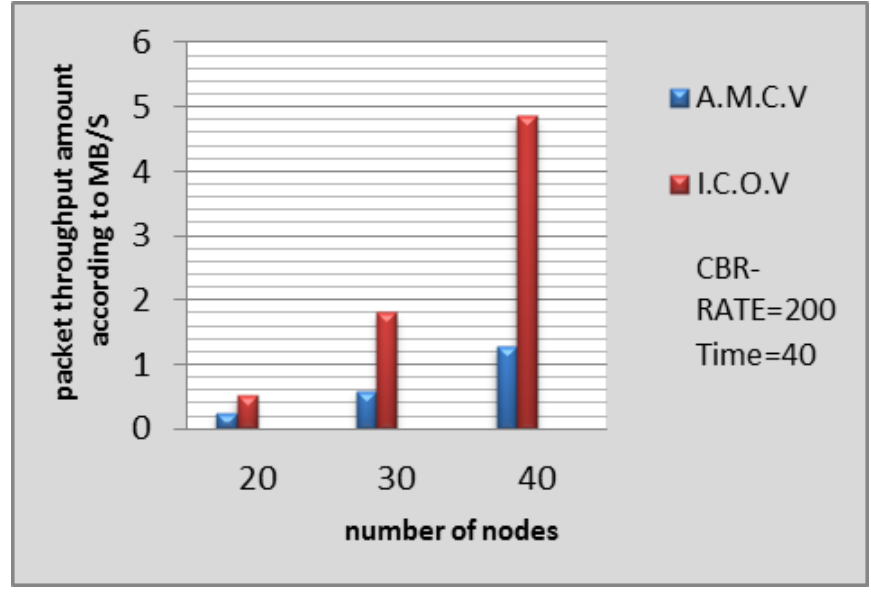

Fig. 10. Packet throughput of decision-making amount with different priority for AMCV and ICOV.

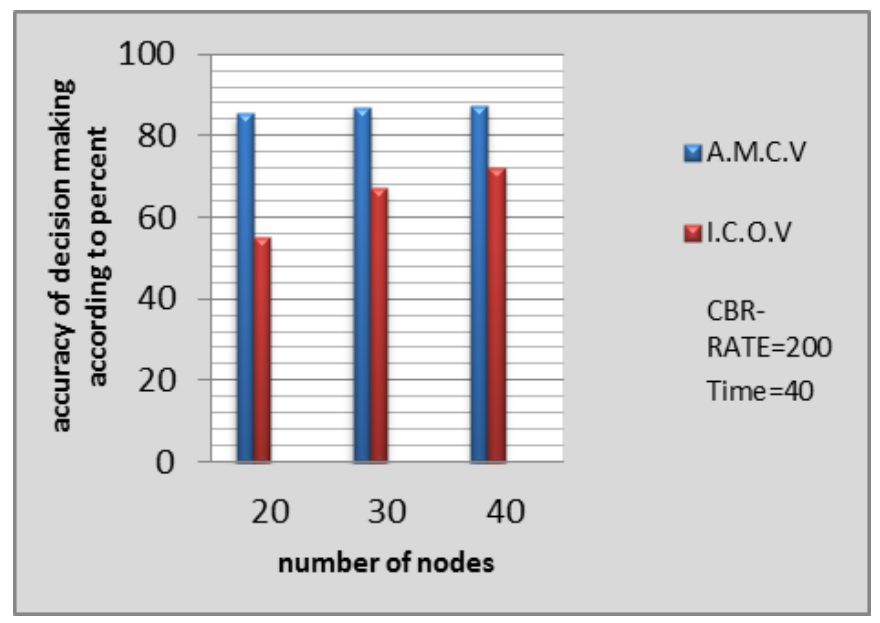

Fig. 11. Accuracy of decision making with different priority for ICOV and AMCV method.

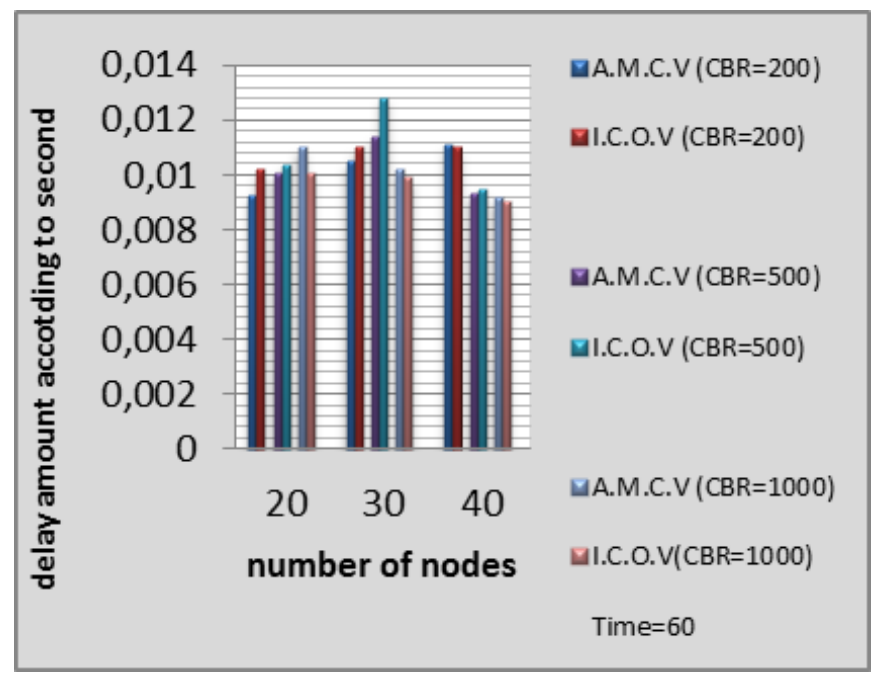

Fig. 12. Latency of AMCV method comparing to ICOV method.

in the network, and at a certain time period and constant traffic rate, the accuracy of decision-making is relatively high. The average shown in Fig. 12 indicates that delay has $0.5 \%$ to $1 \%$

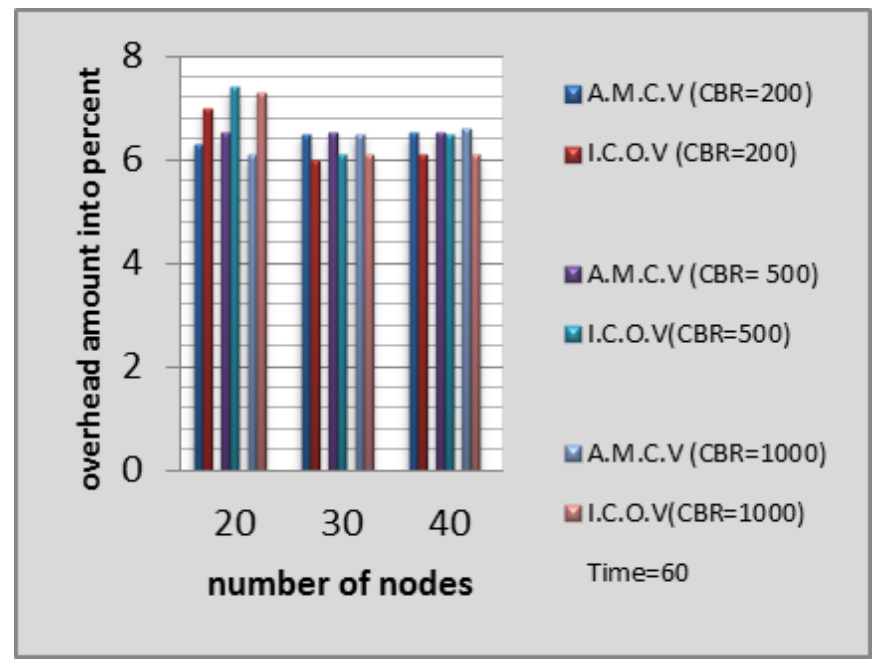

Fig. 13. Overhead amount of AMCV method comparing to ICOV method.

improvement compared to ICOV. Considering the low traffic rate, AMCV delay with different number of nodes, is less than or almost equal to ICOV. Having higher traffic rate on the network and more number of nodes, the delay amount gets closer to ICOV. Fig. 13 indicates that AMCV overhead is at least $0.5 \%$ and at most $1 \%$ lower than ICOV. This is because that the overhead is reduced by clustering in AMCV. However, increasing the number of nodes and traffic rate raises the overhead of AMCV due to the processing overhead of clustering.

In this section, in order to evaluate the proposed method $\mathrm{AMCV}$, some parameters such as type of nodes queuing, protocol type, type of sent packets and packet size are considered. Also, different scenarios are designed in order to study the accuracy of decision making and delay, overhead, and data packets throughput in the network. The results of experiments indicated at least $6 \%$ and at most $85 \%$ improvements in decision making accuracy compared to similar method ICOV. On the other hand, due to the overhead caused by clustering of nodes in the network, the overhead is slightly increased. The delay in the network for AMCV was somehow close to ICOV, and had a slight improvement in low traffic rate and low number of nodes. Also, AMCV had fewer packets' overhead which represents its tolerance in congestion conditions.

\section{CONCLUSION}

With regard to the uncertainty of some nodes in vehicular ad-hoc networks that are attempting to send false information, the proposed method in this paper, AMCV, aimed to improve the trust level of exchanged information between nodes by increasing the accuracy of decision-making. AMCV considered the basic parameters of VANET nodes such as geographical location, direction, speed, node type and degree of relationship with neighbors. In order to reduce the number of dealing nodes in decision-making and increase the degree of certainty, the clustering procedure is performed only on the nodes close to the event position. The obtained experimental results indicated that AMCV reduced the decision-making time period between 
$7 \%$ and $40 \%$ compared to similar method ICOV. It also improved the accuracy of decision-making between $6 \%$ and $85 \%$.

The network operational overhead of AMCV is almost same as ICOV; however in high traffic rate conditions, the overhead of AMCV is around $1 \%$ higher than ICOV which is negligible. The results of throughput represent that, if the execution time gets longer, and having more number of nodes on the network, AMCV is able to act better than ICOV. There is a trade-off between the delay of message transfers and the operational overhead on the network. By increasing nodes in the network environment or reducing the traffic rate on the network data packets, the delay of AMCV is reduced at most $0.5 \%$ compared to ICOV. But when both the number of nodes and traffic rate are increased, the delay of AMCV is increased due to higher clustering overheads.

\section{REFERENCES}

[1] J. Zhang, "A survey on trust management for vanets", in Proc. IEEE Int. Conf. Advanced Information Networking and Applications (AINA), pp. 105-112, March, 2011

[2] A. Fonseca and T. Vazao, "Applicability of position-based routing for VANET in highways and urban environment", Journal of Network and Computer Applications, vol. 36, no. 3, pp. 961-973, 2013.

[3] A. Renuga, T. Rajendran, and B. Suganya, "Collaborative Vehicular Content Dissemination Using Mobility Models and GPSR", Int. J. Emerging Trends in Electrical and Electronics (IJETEE), vol. 2, no. 4, pp. 13-17, 2013.

[4] J. Zhang, "Trust management for VANETs: challenges, desired properties and future directions", International Journal of Distributed Systems and Technologies (IJDST), vol. 3, no. 1, pp. 48-62, 2012.

[5] S. DasGupta, R. Chaki, and S. Choudhury, "TruVAL: trusted vehicle authentication logic for VANET", in Advances in Computing, Communication, and Control, Springer Berlin Heidelberg, pp. 309-322, 2013.

[6] T. Yang, W. Xin, L. Yu, Y. Yang, J. Hu, and Z. Chen, "MisDis: An Efficent Misbehavior Discovering Method Based on Accountability and State Machine in VANET", in Web Technologies and Applications, Springer Berlin Heidelberg, pp. 583-594, 2013.

[7] A. Benaidja, S. Moussaoui, and F. Nait-Abdesselam, "An optimal broadcast of warning messages in vehicular ad hoc networks", International Journal of Computer and Information Technology (IJCIT), vol. 2, no. 5, pp.986-992, 2013

[8] A. Ltifi and A. Zouinkhi, "A Trust Management System Through Ambient Communication for VANET", International Journal of Informatics and Communication Technology (IJ-ICT), vol. 2, no. 2, pp.71-78, 2013

[9] Y. Wang and M.P. Singh, M.P., "Formal Trust Model for Multiagen Systems", in IJCAI vol. 7, pp. 1551-1556, 2007.

[10] Z. Huang, S. Ruj, M.A. Cavenaghi, M. Stojmenovic, and A. Nayak, "A social network approach to trust management in VANETs", Peer-to-Peer Networking and Applications, vol. 7, no. 3, pp.229-242, 2014.

[11] M. Raya, R. Shokri, and J.P. Hubaux, "On the tradeoff between trust and privacy in wireless ad hoc networks", in Proc. third ACM conference on Wireless network security, pp. 75-80, 2010.

[12] S. Jaiswal and D.S. Adane, "Hybrid Approach for Routing in Vehicular Ad-hoc Network (VANET) Using Clustering Approach", International Journal of Innovative Research in Computer and Communication Engineering, pp.2320-9798, 2013.

[13] S. Vodopivec, J. Bester, and A. Kos, "A survey on clustering algorithms for vehicular ad-hoc networks", in Proc. 35th Int. Conf. Telecommunications and Signal Processing (TSP), pp. 52-56, 2012

[14] C. Shea, Aprove: A stable and robust vanet clustering scheme using affinity propagation, Doctoral dissertation, University of Toronto, 2009.

[15] N. Maslekar, M. Boussedjra, J. Mouzna, and H. Labiod, "A stable clustering algorithm for efficiency applications in VANETs", in Proc. 7th Int. Conf. Wireless Communications and Mobile Computing Conference (IWCMC), pp. 1188-1193, 2011.

[16] K. Wang, J. Zhang, D. Li, X. Zhang, and T. Guo, "Adaptive affinity propagation clustering", arXiv preprint arXiv:0805.1096, 2008.
[17] R.T. Goonewardene, F.H. Ali, and E.L.I.A.S. Stipidis, "Robust mobility adaptive clustering scheme with support for geographic routing for vehicular ad hoc networks", IET Intelligent Transport Systems, vol. 3, no. 2, p.148-158, 2009.

[18] A. Koulakezian, "Aspire: Adaptive service provider infrastructure for vanets", Doctoral dissertation, University of Toronto, 2011.

[19] E. Dror, C. Avin, and Z. Lotker, "Fast randomized algorithm for hierarchical clustering in vehicular ad-hoc networks", in Proc. 10th IFIP Annual MediterraneanAd Hoc Networking Workshop (Med-Hoc-Net), pp. 1-8, 2011

[20] H. Su and X. Zhang, "Clustering-based multichannel MAC protocols for QoS provisionings over vehicular ad hoc networks", IEEE Trans Vehicular Technology, vol. 56, no. 6, pp.3309-3323, 2007.

[21] Z.Y. Rawshdeh and S.M. Mahmud, "Toward strongley connected clustering structure in vehicular ad hoc networks". In Proc. IEEE 70th Vehicular Technology Conference Fall (VTC 2009-Fall), pp. 1-5, 2009.

[22] Z.Y. Rawashdeh and S.M. Mahmud, "A novel algorithm to form stable clusters in vehicular ad hoc networks on highways", EURASIP Journal on Wireless Communications and Networking, vol. 2012, no. 1, pp.1-13, 2012.

[23] M.S. Almalag and M.C. Weigle, "Using traffic flow for cluster formation in vehicular ad-hoc networks", in Proc. 35th Conf. Local Computer Networks (LCN), pp. 631-636, 2010.

[24] S.S. Wang and Y.S. Lin, "PassCAR: A passive clustering aided routing protocol for vehicular ad hoc networks", Computer Communications, vol. 36, no. 2, pp. 170-179, 2013. 